\title{
Minimizing interference in automotive radar using digital beamforming
}

\author{
C. Fischer ${ }^{1}$, M. Goppelt ${ }^{2}$, H.-L. Blöcher ${ }^{1}$, and J. Dickmann ${ }^{1}$ \\ ${ }^{1}$ Daimler AG, Wilhelm-Runge-Straße 11, 89013 Ulm, Germany \\ ${ }^{2}$ University of Ulm, Institute of Microwave Techniques, Albert-Einstein-Allee 41, 89069 Ulm, Germany
}

\begin{abstract}
Millimetre wave radar is an essential part of automotive safety functions. A high interference tolerance, especially with other radar sensors, is vital. This paper gives an overview of the motivation, the boundary conditions and related activities in the MOSARIM project funded by the European Union and concerned with interference mitigation in automotive radars. Current and planned activities considering Digital Beamforming (DBF) as a method for interference mitigation are presented.
\end{abstract}

\section{Introduction: future automotive scenarios}

A global trend or "megatrend" to be expected in the midterm future is increasing urbanization and thus the growth of megacities. In the process of this development, in addition to the increasing number of people living in cities, there will also be more cars in the streets (an illustrative example is given in Fig. 1). Recent studies also expect a growing number of cars in emerging countries (cf. IEA, 2008). Automotive safety is an essential requirement and vital necessity. There is another implication for the increasing number of cars equipped with safety functions based on radar technology: As the car density and absolute number of cars using radar increase, the chance of two radar sensors interacting in some way also increases. In anticipation of this development, the EU Seventh Framework Programme MOSARIM (see MOSARIM Webpage, 2010) was initiated by a number of OEMs, Tier 1 and scientific institutes. This project has three major goals:
- to identify and analyze the mechanisms of interference between automotive radar sensors

- and subsequently to derive guidelines to minimize the probability of interference in future sensor generations.

The project commenced in January 2010 and will end in December 2012. A workshop will be held where the final results will be presented to the public.

This paper is structured as follows: First a short estimation of the future development of radar equipped cars is presented. Then, Digital Beamforming is briefly presented and the methods considered for interference mitigation in Wirth (2001) are described. Finally, the simulation environment is introduced and results presented. The paper concludes with a summary and an outlook.

\section{Digital beamforming}

Digital Beamforming (DBF) is a signal processing method with growing importance. As semiconductor technology advances, more and more parts of a radar system can actually be computed in software rather than realized in hardware. Key components for DBF are the analog-digital converters (ADC) and the digital signal processing element realized in an FPGA, DSP or an ASIC. Compared with currently widely used automotive radar sensors, no moving parts are necessary to measure the incident angle of a signal. Complicated high frequency hardware like RF phase shifters may also be omitted because of the early digitization of the signals. This makes DBF an important candidate for cost reduction while at the same time increasing flexibility and reliability. 


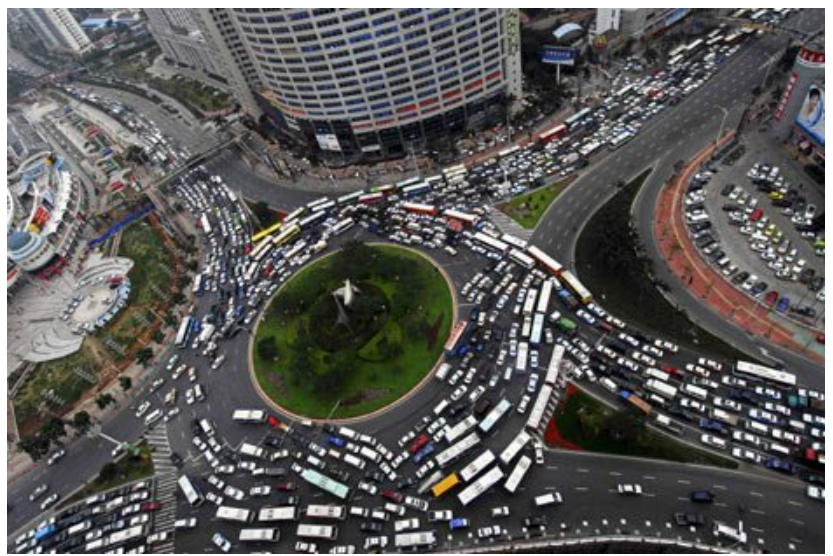

Fig. 1. Inner city traffic scenarios with a large number of radar sensors like this will be an increasing challenge for radar sensors and implemented interference mitigation techniques in the future.

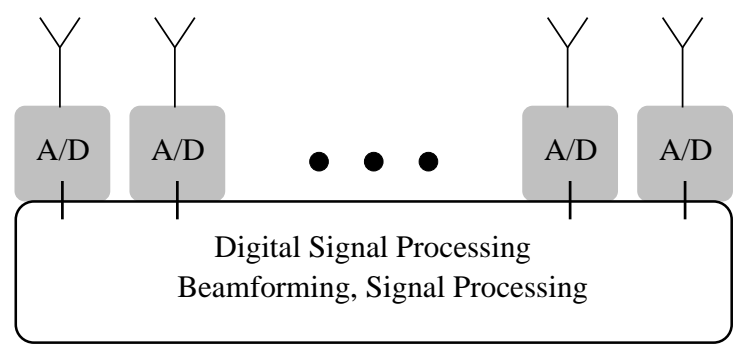

Fig. 2. The basic principle of Digital Beamforming: The received signals will be sampled close to the antenna and processed in parallel.

The basic principle of DBF is simple (see Fig. 2): A fixed number of antennas $N$ at different positions receive in parallel. This can be regarded as spatial sampling that results in a number of signals:

$s_{i}(t) \quad$ with $\quad i=1 \ldots N$.

After reception and downconversion the signals are sampled in time by the ADCs:

$x_{i}[k]=s_{i}(k T) \quad$ with $\quad T=\frac{1}{f_{\text {Sample }}}, k \in \mathbb{N}$.

Being in the digital domain, the signals $x_{i}[k]$ can be processed with great flexibility. The best known application in DBF is the estimation of the incident angle (or direction) of arrival (AoA or DoA) of a target. This can be done for a linear antenna array simply by computing a discrete Fourier tranform (DFT) across the individual elements of the array. Because of this, the term angular spectrum is often used. In matrix notation this can be expressed simply by

$\mathbf{x}_{a}[k]=\mathbf{A} \mathbf{x}[k]$,

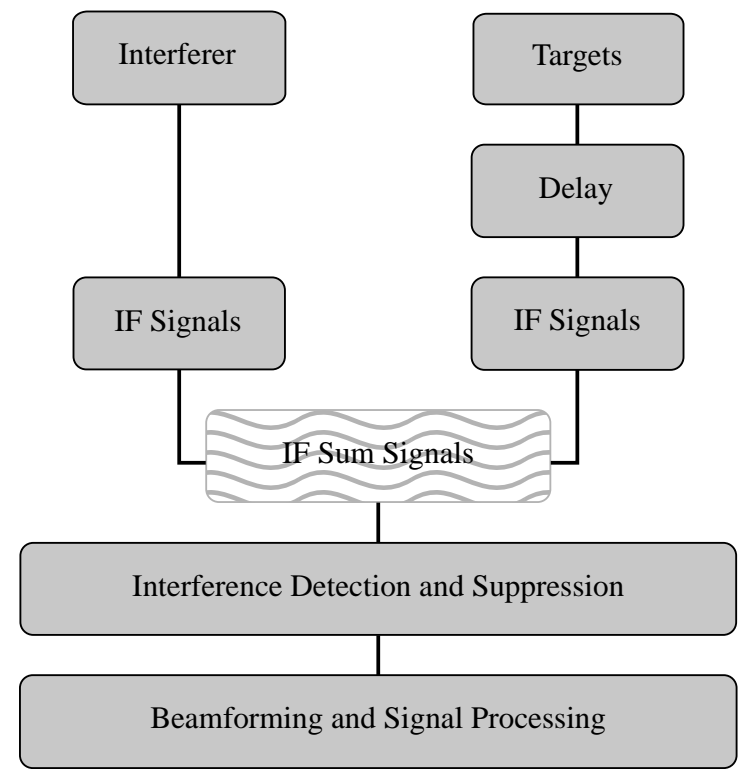

Fig. 3. Simplified block diagram of the implemented simulation environment.

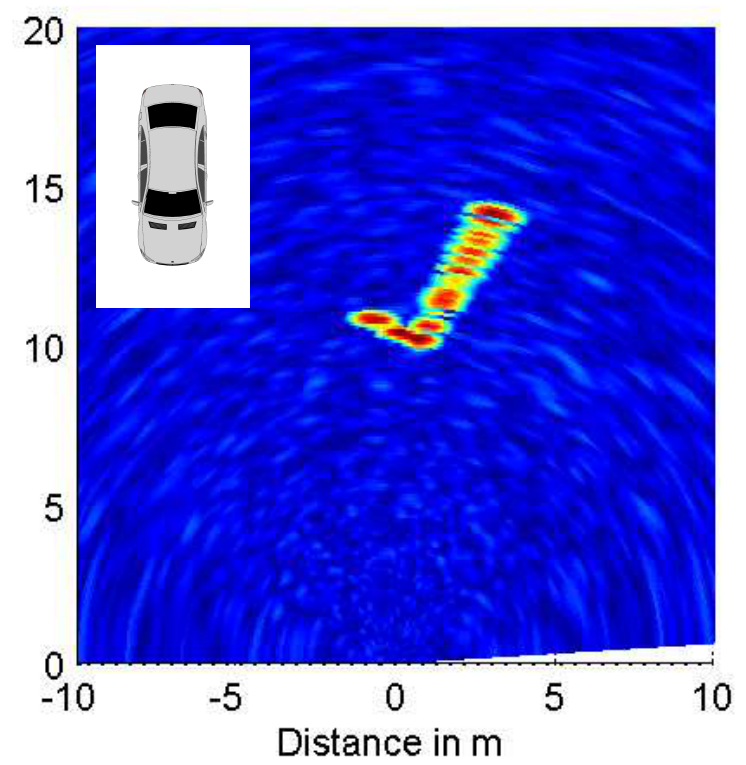

Fig. 4. An example of a simulation result: The basic simulation scenario without any interference but with additive white Gaussian noise (red $=$ high amplitude, blue $=$ low amplitude).

with A being the transformation matrix. Using this formalism it is possible to stack different linear processing steps into this matrix notation. This form is also advantageous for calculating transformations other than the DFT, for which the FFT already provides a fast algorithm. For matrix 


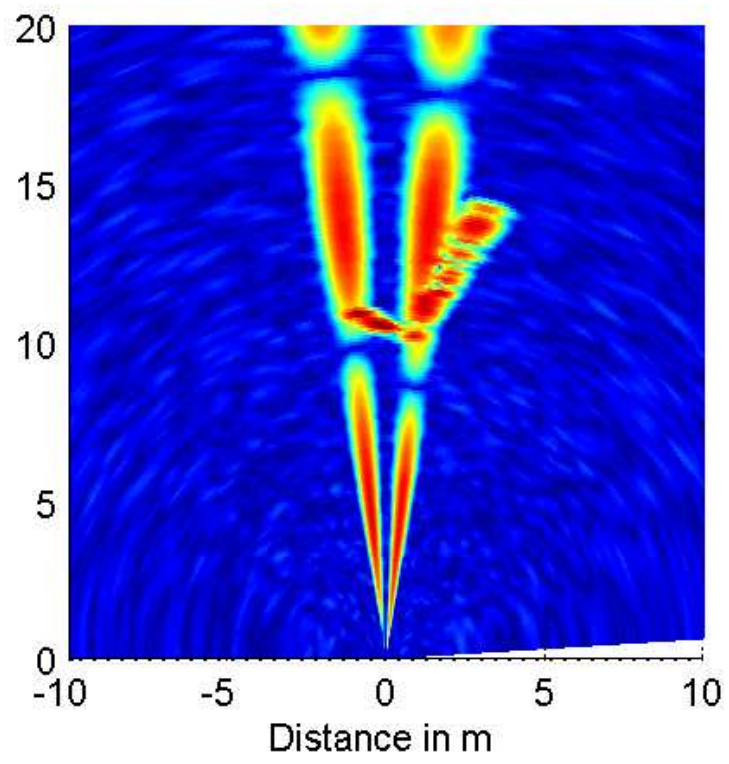

Fig. 5. Simulation result: The scenario with interference and additive white Gaussian noise but without any mitigation technique applied (red $=$ high amplitude, blue = low amplitude).

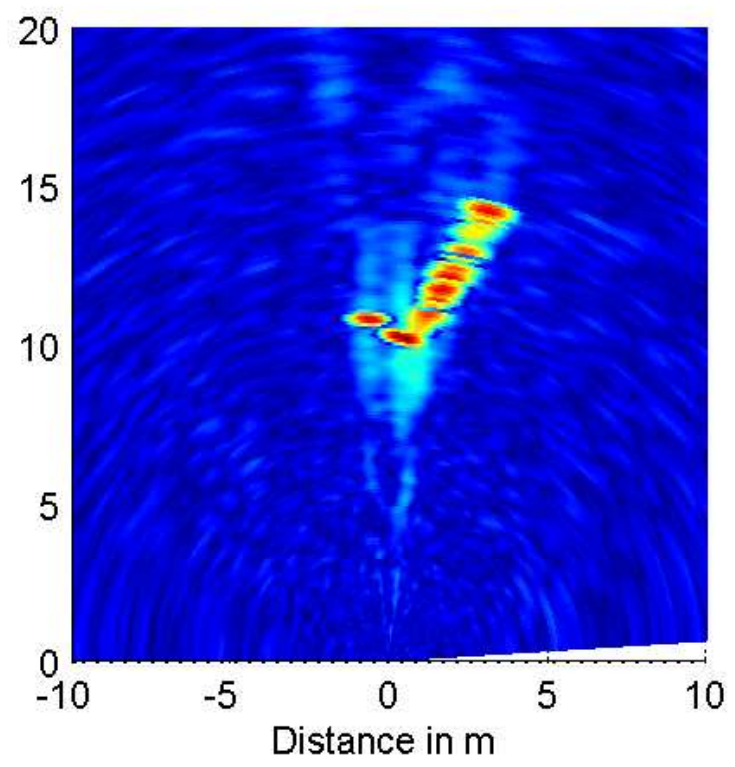

Fig. 6. Simulation result: The scenario with interference and additive white Gaussian noise (red = high amplitude, blue = low amplitude). The samples affected by the interference are zeroed.

multiplication, fast algorithms exist that can be used for implementation on the desired hardware platform, such as an FPGA, for example.

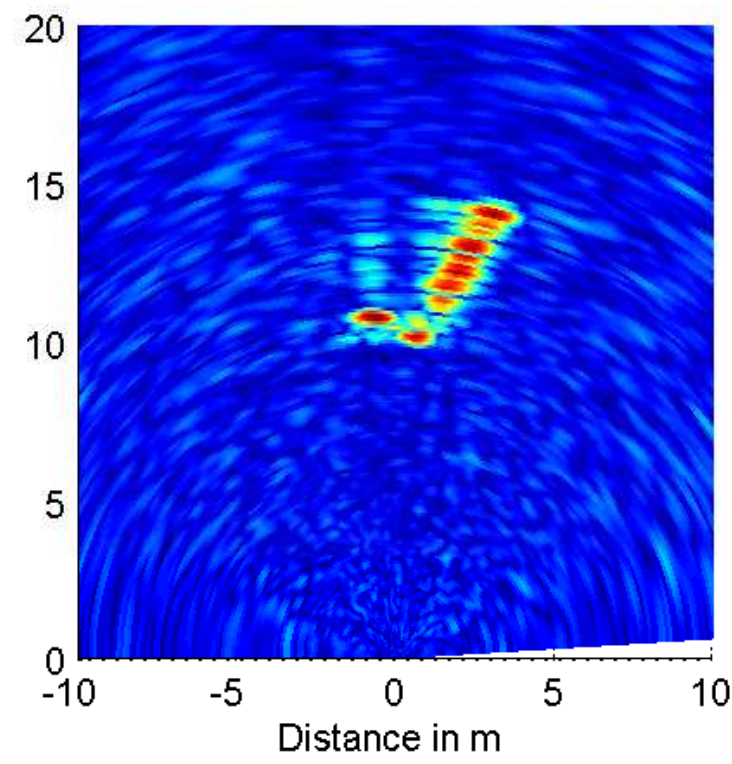

Fig. 7. Simulation result: The scenario with interference and additive white Gaussian noise (red = high amplitude, blue = low amplitude). The interference is canceled with LSMI.

\section{Interference identification and suppression}

Goppelt et al. (2010) showed that interference in FMCW radar systems can have severe impact on the IF signals. Although no influence on the object level has been observed so far, the signal to noise ratio degrades. To reduce this effect before the target acquisition step in the radar sensor, the interference should be suppressed as early as possible in the signal processing chain. In this paper, the focus is on short time interference with high amplitude relative to the wanted signals. Due to the architecture of FMCW signals, this kind of interference is most likely to occur in real driving situations, for example, when two radar equipped cars drive towards each other on a straight road. Considering ramp ups followed by ramp downs, which are typical in standard FMCW systems, there has to be a point in time where these ramps intersect and where the above mentioned interference pattern occurs.

The identification of the interference is the first step to its mitigation. In the case considered here, the use of the spiky time domain signal characteristics is obvious. The energy of the signals

$e_{i}[k]=x_{i}[k]^{2}$

convolved with a moving average kernel is therefore considered. When the average energy in the considered timeframe rises above a given limit, defined by the scaled mean energy of the complete signal, an interference is declared. Since a DBF system with several receive channels is being consid- 
ered, this procedure is applied to all channels separately. After the detection step, the length of the interference over all channels is defined by the first and the last detection.

From these identified interference sequences $y_{i}[k]$, the covariance matrix $\mathbf{Q}$ must now be determined. It needs to be estimated based on $S$ samples or snapshots of the received signals:

$\hat{\mathbf{Q}}=\frac{1}{S} \sum_{S=1}^{S} \mathbf{y}_{S} \cdot \mathbf{y}_{S}^{*}=\frac{1}{S} \mathbf{Y} \mathbf{Y}^{*}$.

A noise matrix is added to reduce the influence of fluctuations of small eigenvalues:

$\hat{\mathbf{Q}}=\frac{1}{S} \mathbf{Y} \mathbf{Y}^{*}+\alpha \cdot \mathbf{I}$.

This also ensures the covariance matrix to be non-singular and therefore invertible. After this preparation, the interference is suppressed by computing

$\hat{\mathbf{x}}[k]=\mathbf{A}^{*} \hat{\mathbf{Q}}^{-1} \mathbf{x}[k]$.

The reconstructed angular spectrum $\hat{x}_{i}[k]$ can then be further processed. This method is known as loaded sample matrix inversion (LSMI). One point to be mentioned is that a small part of the desired signal is also removed due to the very simple algorithm for estimating the covariance matrix. The interference suppression is not only a function of the length of the identified interference, but also of the interference to signal ratio. Note that if the interference is too weak, the suppression makes no more sense since the interference power is spread across the whole range profile and therefore has little influence on reasonably strong targets. Quantitative comparisons on that will be the subject of further research.

\section{Simulation environment and first results}

A simulation chain has been implemented in MATLAB ${ }^{\circledR}$ to simulate the effects of different interference scenarios on a DBF radar. This simulation can resemble an arbitrary FMCW radar with multiple receiving antennas. For now the individual blocks are assumed to be completely linear, but some quantization effects are already implemented. The block diagram in Fig. 3 shows the basic elements. In the first step, the targets are defined and the propagation delay to each of the the receiving antennas is calculated. The resulting intermediate frequency (IF) is calculated for each target from these delays and a velocity property of the targets and then combined with the interference signals generated separately. The result of this accumulation is a sampled multichannel IF signal. For the simulations shown in this paper, a value of 8192 samples per ramp has been considered, but this value can be changed to any value necessary for an application. Although we consider static scenarios here, it is also possible to simulate dynamic scenarios.
The scenario used for the interference tests is displayed in Fig. 4. The L-shape was selected as the target because it can be interpreted as the reflection pattern of a car. In the simulation, it was represented by a number of point scatterers that were positioned accordingly. The interferer was placed in front of the L-shape. The scenario can therefore be considered as a car equipped with a similar sensor to the simulated one, driving towards the victim at a certain angle.

In Figs. 5 to 7, some first results of the mentioned algorithm are displayed. For comparison, what happens when the affected bins are simply set to zero (see Fig. 6) was also simulated. It can clearly be seen that this procedure results in significant distortion in the range direction, whereas the LSMI displayed in Fig. 7 results in a slight distortion in the angular direction. Further techniques that use extrapolation or intrapolation to reconstruct the part of the signal affected by interference already exist. These will also be implemented in the setup and tested for practical use. In subsequent steps, the implemented algorithms will need to be applied to real data from an automotive sensor to show their usefulness in practice.

\section{Conclusions}

In the future, interference mitigation will become an important issue in automotive radar. Using a DBF simulator the applicability of well-known interference mitigation techniques could be displayed. Analysis will continue with the use of data from real sensors and the implementation of more sophisticated techniques. In the long term these techniques will be implemented on real hardware to undergo real world tests. The complexity of the algorithms and the possibility for realization on the FPGA of the existing platform will therefore also be an issue.

Acknowledgements. The research leading to these results has received funding from the European Community's Seventh Framework Programme (FP7/2007-2011) under grant agreement no 248231 - MOSARIM Project.

\section{References}

Goppelt, M., Blöcher, H.-L., and Menzel, W.: Automotive radar -investigation of mutual interference mechanisms, Adv. Radio Sci., 8, 55-60, doi:10.5194/ars-8-55-2010, 2010.

IEA (International Energy Agency): World Energy Outlook, 2008. MOSARIM Webpage: http://www.mosarim.eu, last access: 17 November 2010.

Wirth, W.-D.: Radar techniques using array antennas, IET radar, sonar, navigation and avionics series 10 , edited by: Steward, N. and Griffith, $\mathrm{H}$. The institution of engineering and technology, London, 2001 